\title{
Cardiovascular Outcomes in Renal Transplant Recipients: Feasibility and Clinical Role of 2D Speckle Tracking to Assess Myocardial Function
}

\author{
Laura Stefani ${ }^{1, *}$, Gianni Pedrizzetti ${ }^{2}$, Stefano Pedri ${ }^{3}$, Enrico Minetti ${ }^{4}$, Marco Mandoli ${ }^{1}$, \\ Benedetta Tosi ${ }^{1}$ and Giorgio Galanti ${ }^{1}$ \\ 1 Sports Medicine Center, Clinical and Experimental Department, University of Florence, 50100 Florence, Italy; \\ marco.mandoli@yahoo.it (M.M.); benedetta.tosi@unifi.it (B.T.); giorgio.galanti@unifi.it (G.G.) \\ 2 Engineering and Architectural Department, University of Trieste, 34121 Trieste, Italy; giannip@dia.units.it \\ 3 ESAOTE-Italy, 50100 Florence, Italy; stefano.pedri@esaote.com \\ 4 Division of Nephrology, University Hospital Careggi, 50100 Florence, Italy; minettie@aou-careggi.toscana \\ * Correspondence: laura.stefani@unifi.it; Tel.: +39-347-768-9030; Fax: +39-055-794-9902
}

Academic Editor: Giuseppe Musumeci

Received: 13 January 2016; Accepted: 22 February 2016; Published: 2 March 2016

\begin{abstract}
Left ventricular (LV) function is normally improved after renal transplant; however, cardiovascular mortality remains elevated. Moderate physical activity has a positive impact on myocardial function; however, few data are available about the role of 2D echocardiography (2DE) and 2D speckle tracking echocardiography (2DSTE) on renal transplant recipients (RTR). From a large cohort of RTR submitted to a supervised exercise as the prescription program, 10 subjects who were regularly trained were studied for sixth months. They underwent periodically an echo evaluation (ESAOTE MyLab 50), cardiopulmonary test (CPT) and strength test for the lower and upper limbs. The LV function study was completed with the speckle tracking longitudinal strain (Lo Strain) measure calculated by dedicated software (XStrain-Esaote) at the end of the protocol. All of the cardiovascular parameters were normal: the ejection fraction (EF) increased significantly (from $62.7 \pm 4$ to $67.2 \pm 2.3$ with $p<0.05$ ), as well as the anaerobic threshold (15.3 \pm 6.8 to $20.5 \pm 10.1$ with $p<0.05)$. Particularly, the global longitudinal strain (GLS) values were within the normal range $(-19.2 \% \pm 5.1)$, maintaining the physiological gradient from the basal $(-13.2 \pm 4.1 ;-16.5 \pm 5.21)$ to the apex level $(-21 \pm 2.3 ;-25.7 \pm-7.0)$. 2D speckle tracking echocardiography (2DSTE) can be effectively used to confirm the presence of preserved physiological myocardial function in post-renal transplantation subjects submitted to a physical training.
\end{abstract}

Keywords: strain; exercise; renal transplant

\section{Introduction}

It has been widely demonstrated that physical exercise plays an important role in the prevention of metabolic and cardiovascular risk factors [1]. After surgical treatment, solid organ recipients often show metabolic syndrome [2,3], where the risk of possible acute cardiac events and the risk of cardiovascular mortality remains unfortunately high. Improvement in maximal and sub-maximal exercise capacity has been demonstrated through aerobic and resistance activity, particularly in the post-transplant syndrome [4-6]. The maintenance of the left ventricle myocardial function is therefore relevant and mandatory during the exercise as the prescription program. In addition to the traditional echocardiographic parameters, the deformation parameters of the left ventricle (LV) chamber, particularly the longitudinal strain (Lo Strain), are currently used as a noninvasive method to study and to follow the presence of preserved myocardial function $[7,8]$ in the case of regular training. Speckle tracking echocardiography (2DSTE) has already demonstrated its clinical 
potential to investigate LV [9] function. However, a practical limitation to the extensive use of these techniques in routine clinical practice has been the perceived significant variability that exists among brands. Recently, this perception has been overcome after the publication of studies conducted by American Society of Echocardiography (ASE)and European Association Cardiovascular Imaging (EACVI) scientific co-ordination in which inter-vendor variability and the reproducibility have been assessed both on synthetic dataset [10] and "in vivo" testing on patients [11], and normality values for longitudinal strain have been provided in the new ASE guidelines for chamber quantification [12]. However, there are no data available about the possible application of the Lo Strain evaluation in post-transplant syndrome. The present investigation aims to assess the maintenance of the myocardial performance during a short-term personalized mixed exercise training, using the echocardiographic exam and the feasibility of the 2D longitudinal strain application as an additional method to follow the contractility of the heart.

\section{Materials and Method}

\subsection{Population Studied}

Among a cohort of renal transplant recipients, 20 subjects were enrolled into a special exercise program as therapy to control the potential cardiovascular risk factors associated with the post-transplant metabolic syndrome. The group was composed of 12 male and 8 female, aged $51.6 \pm 6.2$, surgically treated at least $6.2 \pm 6.1$ years before the study. The cause of the chronic renal failure (CRF) was polycystic kidney in 6, pyelonephritis in 5, nephroangiosclerosis in 3, Alport syndrome in 2 and glomerulonephritis in 4 cases.

All of the subjects were asymptomatic for acute cardiovascular events, with clinical stability of blood pressure and glycemic levels. They were under pharmacological treatment consisting of cortisone (methylprednisolone) antihypertensives, such as doxazosin, angiotensin converting enzyme (ACE) inhibitors or sartans and platelet aggregation inhibitors and immunomodulators, such as tacrolimus, cyclosporine, everolimus and mycophenolate, without any clinical and echocardiographic evidence of cardiotoxicity. The study was approved by the local ethics committee, and all of the subjects gave informed written consent.

All subjects were submitted to a period of supervised physical exercise for at least 6 months; however, only 14 concluded the program spontaneously, and among them, only 10 subjects were considered eligible for the final evaluation by training analysis due to high quality images. The physical exercise was practiced in a supervised regimen, in a dedicated fitness gym, under the supervision of the motor science specialists.

The exercise program administered was mixed, both aerobic and resistance. The individual level of aerobic exercise was established by a cardio pulmonary exercise test (CPET). It was carried out using a Schiller Cardiovit Ergo-Spiro CS 200 (Schiller AG, Baar, Switzerland) according to the standard Bruce cycle test protocol conducted up to complete exhaustion. Following the recent American College Sports Medicine (ACSM) guidelines [13], the exercise program consisted of $30 \mathrm{~min}$ of aerobic exercise on a stationary bike at the aerobic threshold level. Regarding the resistance exercise, the muscle strength of the upper and lower limb was established following an indirect estimate [14] by the hand grip test and the chair stand test. The first level of resistance exercises, composed of the principal movements performed in absence of additional weight and therefore at low charge, were prescribed. No individual indications were performed for this kind of exercise, as the changes of the muscular component are not one of the study targets. All of the subjects were regularly submitted to an echocardiographic exam in resting conditions, performed at the onset of the study and after 6 months of training. Only at the end of the study was the evaluation of the myocardial function of the 10 renal transplant recipients who were regularly trained completed by the longitudinal strain analysis $\left(\mathrm{S}_{1}\right)$ [15]. The $\mathrm{S}_{1}$ values were compared to a group of healthy subjects, similar to the renal transplant recipients (RTR) for their general characteristics and aged $48.5 \pm 5.2$. They were submitted to the same exercise program 
and for the same time. All of the healthy subjects were free of chronic disease and regularly trained. The aerobic component of the exercise, and therefore, the individual heart rate (HR) for the exercise program, was established focusing on $60 \%$ of the $91 \%$ maximal effort calculated by the ergometric cycling test. The exercise program consisted of $30 \mathrm{~min}$ of aerobic exercise on a stationary bike at the aerobic threshold level. Regarding the control group, considering that the subjects were healthy, the cardiopulmonary test (CPT) was not assumed as mandatory to plan their exercise program. The intensity of the exercise prescribed was therefore established using a cycling ergometric test. It was performed at the maximal effort following the Bruce protocol. The test was conducted with the use of the Borg CR-10 effort scale. The HR, corresponding to an effort of 4 to 5 points of the CR10 effort scale, was identified as the best for their exercise program.

\subsection{Cardiopulmonary Test}

During the CPET, respiratory gas measurements were obtained using Schiller's Cardiovit Ergo-Spiro CS 200 (Schiller AG). Calibrations of the flow transducer and gas analyzers were performed daily. The transducer calculates several parameters with a mouthpiece for detecting breath-by-breath registrations of oxygen uptake $\left(\mathrm{VO}_{2}\right)$, expired $\mathrm{CO}_{2}\left(\mathrm{VCO}_{2}\right)$, minute ventilation (VE) and respiratory exchange ratio (RER). The exercise test was performed following a modified Bruce protocol [16] using a cycle ergometer. The protocol was appositely modified to guarantee a progressive enhancement of effort. The first workload was $25 \mathrm{~W}$, increasing $25 \mathrm{~W}$ every $2 \mathrm{~min}$ up to subjective exhaustion. The cycling test was conducted using a Schiller Cardiovit Ergo-Spiro CS 200 cycle ergometer (Schiller AG). During exercise, the rate per minute was constant at 70 repetitions $/ \mathrm{min}$. The test was conducted up to complete exhaustion. Aerobic capacity, distinguished by the aerobic threshold, anaerobic threshold and $\mathrm{VO}_{2}$ peak level, was obtained from CPET. For the aerobic threshold (AerT) and anaerobic threshold (AT), two points that were discontinuous during the incremental effort, and the $\mathrm{VO}_{2}$ peak level at maximal effort were considered, respectively. As the literature reports, these are normally identified either in the ventilator curve response or in the lactate plasma level curve [17]. Only the former, corresponding to the blood lactate level of around 2 to $4 \mathrm{mmol}$, is not routinely used in evaluating athletes' performance. The transition from aerobic to anaerobic metabolism, and therefore, from the AerT to AT value, has been confirmed in this investigation by two blood samples from the antecubital fossa, to determine the achievement of the aerobic target range.

The heart rate (HR) of each of the subjects, corresponding to the intensity of the exercise around the degree of the matched METs (metabolic equivalent) and to the AerT, particularly at moderate intensity of physical exercise, was used to establish the intensity of the exercise individually prescribed. In order to better identify the intensity of effort, CPET was integrated with the adult OMNI (omnibus) scale of perceived exertion, such as Borg CR-10 [18].

\subsection{Echocardiographic Examination}

The echocardiogram was performed by qualified cardiologists trained in sonography, using a MyLab 50 echocardiograph (Esaote, Italy), equipped with a 2.5-MHz transthoracic echocardiographic probe. According to the American College of Cardiology (ACC) and American Heart Association (AHA) guidelines [19], the echocardiographic measurements of the left ventricle (LV) chamber were obtained from the parasternal long-axis view. They included LV end-systolic and end-diastolic diameters (LVEDd, LVESd), interventricular septum (IVS), posterior wall thickness (PW) and the ejection fraction (EF\%), calculated according to the formula (LVEDD-LVESD/LVEDD, for which volumes are substituted by diameters. Doppler analysis was used to measure the diastolic variables in the presence of a stable Rate of $R$ wave(RR )interval at the Elettrocardiographic (ECG) monitor and in three different, but sequential measurements from the four-chamber view. They consisted of transmitral flow of $\mathrm{E}$ wave and A wave peak velocities, isovolumetric relaxation time (IVRT) and deceleration time (DTc) and the E/A ratio. The degree of severity of the insufficiency, described as the extent of the regurgitant jet on a 0 to $4+$ scale, was assessed using the color-flow mapping 
method from the four-chamber view, according to the ACC/AHA guidelines [19]. The index of left ventricular mass (LVMI) and left ventricular hypertrophy (LVH) was calculated according to the Devereux formula [20]. Aortic valve morphology was examined from the parasternal long- and short-axis views. The evaluation of the aorta was completed by measuring the ascending aortic tract at four different levels: aortic annulus (Aoan), sinuses of Valsalva (Aosv), sino-tubular junction (Aostj) and proximal ascending aorta (PAA) at $1 \mathrm{~cm}$ from the sino-tubular junction, in order to rule out any aneurysmal dilatation, which would represent a contraindication to enrolment. The eventual presence of pathologic aortic valve stenosis or regurgitation was assessed by continuous-wave Doppler using the apical 4-chamber view.

The echocardiographic examination was completed with the evaluation of other possible anatomic or hemodynamic abnormalities, such as the presence of other valvular regurgitation and with the calculation of pulmonary pressure, interventricular or atrial septal defects.

\subsection{Strain Analysis by Speckle Tracking Model}

The 2D images of four-chamber views were post-processed with X-Strain software to provide an angle-independent tool for the evaluation of velocities and strain. This software allows automatic evaluation of both endocardial and sub-endocardial edges from 2D B-mode echocardiographic clips. Strain analysis by speckle tracking is independent of translational motion and the tethering effects of the nearby regions, and it thus allows uniformity of measurements through the normal LV myocardium.

The endocardial border is drawn by the operator in a four-chamber view on a single frame from one annulus to another; the first and last points delineate the mitral plane. Because the global longitudinal strain (GLS) has better reproducibility than other deformation indices [8], this evaluation has been preferred compared to the others parameters, at least for the first approach in this kind of patient. The LV-GLS, average Lo Strain and LPSS at the basal and mid-apical segments of the lateral wall (LW) of LV and the IVS were calculated in all of the subjects captured at rest.

\subsection{Muscle Strength and Functional Abilities}

Muscle strength and resistance for the upper and lower limbs were established following an indirect estimate [9] by the chair stand test to measure the number of repetitions, and the hand grip test consists of a muscle strength test using a hand gripper. The tests were considered exclusively to exclude the possibility of a poor muscular force as a potential contraindication to the eventual adhesion to the protocol.

\section{Results}

All 10 renal transplant recipients enrolled and who were regularly training were considered suitable for the myocardial strain acquisition. During the protocol, no arrhythmic events were observed among the subjects enrolled. None of the subjects presented cardiovascular symptoms, and therefore, no other subjects were excluded from the study for such a reason. All data, expressed as mean SD, were normal in the two phases of observation (Tables 1 and 2). 
Table 1. Cardiovascular metabolic parameters of the renal transplant recipients.

\begin{tabular}{ccccc}
\hline $\begin{array}{c}\text { Number 10 Transplant } \\
\text { Recipients }\end{array}$ & Parameters & T0 & T6 & $p$ \\
\hline \multirow{3}{*}{ Aerobic Threshold } & $\mathrm{VO}_{2}\left(\mathrm{mLO}_{2} / \mathrm{kg} / \mathrm{min}\right)$ & $11.5 \pm 7.8$ & $11.4 \pm 5.4$ & $\mathrm{NS}$ \\
& $\mathrm{HR}(\mathrm{bpm})$ & $105 \pm 23.2$ & $101.7 \pm 14.1$ & $\mathrm{NS}$ \\
& Lactate $(\mathrm{mmol})$ & $3.5 \pm 1.4$ & $3.6 \pm 0.9$ & $\mathrm{NS}$ \\
\hline \multirow{3}{*}{ Anaerobic Threshold } & $\mathrm{VO}_{2} \mathrm{max}\left(\mathrm{mLO}_{2} / \mathrm{kg} / \mathrm{min}\right)$ & $15.3 \pm 6.8$ & $20.5 \pm 10.1$ & $<0.05$ \\
& $\mathrm{HR}(\mathrm{bpm})$ & $119.4 \pm 19.7$ & $118.6 \pm 19.8$ & $\mathrm{NS}$ \\
& $\mathrm{Load}(\mathrm{watt})$ & $67.5 \pm 45.9$ & $77.2 \pm 67.0$ & $\mathrm{NS}$ \\
\hline & $\mathrm{VO}_{2}(\mathrm{mLO} / \mathrm{kg} / \mathrm{min})$ & $22.6 \pm 8.2$ & $25.4 \pm 13.0$ & $\mathrm{NS}$ \\
$\mathrm{VO}_{2}$ Peak & $\mathrm{HR} \mathrm{max} \mathrm{(bpm)}$ & $140.1 \pm 24.1$ & $135.4 \pm 20.2$ & $\mathrm{NS}$ \\
& $\mathrm{Load} \max (\mathrm{watt})$ & $112.5 \pm 61.6$ & $111.3 \pm 62.4$ & $\mathrm{NS}$ \\
\hline
\end{tabular}

T0: Time0; T6: after sixth months HR: heart rate; $\mathrm{VO}_{2}$ : oxygen consumption; Lactate: lactate plasmatic concentration; NS: not significant.

Table 2. 2D-echocardiographic parameters of the renal transplant recipients.

\begin{tabular}{|c|c|c|c|}
\hline N10 Transplant Recipients & T0 & T6 & $p$ \\
\hline Ao $(\mathrm{mm})$ & $33.4 \pm 4.0$ & $33.1 \pm 3.7$ & NS \\
\hline Left Atria (mm) & $36.7 \pm 4.6$ & $36.1 \pm 3.9$ & NS \\
\hline IVS (mm) & $9.5 \pm 0.9$ & $9.4 \pm 0.7$ & NS \\
\hline PW (mm) & $9.0 \pm 0.6$ & $9.3 \pm 1$ & NS \\
\hline $\operatorname{LVdD}(\mathrm{mm})$ & $50.5 \pm 3.8$ & $49.2 \pm 3.2$ & NS \\
\hline $\mathrm{LVsD}(\mathrm{mm})$ & $30.2 \pm 2.6$ & $30.1 \pm 2.9$ & NS \\
\hline $\mathrm{EF} \%$ & $62.7 \pm 3$ & $67 \pm 2.3$ & 0.02 \\
\hline $\mathrm{RV}(\mathrm{mm})$ & $23.2 \pm 0.9$ & $23.6 \pm 1$ & NS \\
\hline $\mathrm{CMI}(\mathrm{g} / \mathrm{m} 2)$ & $107 \pm 20.8$ & $104.7 \pm 1.4$ & NS \\
\hline $\mathrm{T} / \mathrm{R}$ & 0.38 & 0.38 & NS \\
\hline E & $72.5 \pm 16$ & $69.9 \pm 11.7$ & NS \\
\hline A & $72.9 \pm 19$ & $68.4 \pm 11.4$ & NS \\
\hline $\mathrm{E} / \mathrm{A}$ & $2.05 \pm 2.4$ & $1.02 \pm 0.2$ & NS \\
\hline IVRT & $76.8 \pm 7.6$ & $74.5 \pm 10.4$ & NS \\
\hline DTc & $201.6 \pm 48.1$ & $194 \pm 40$ & NS \\
\hline $\mathrm{E}^{1}$ & $8.5 \pm 1.9$ & $7.8 \pm 2.4$ & NS \\
\hline $\mathrm{A}^{1}$ & $9.8 \pm 3.4$ & $9.2 \pm 2.9$ & NS \\
\hline$E / E^{1}$ & $9 \pm 2.7$ & $9.4 \pm 2$ & NS \\
\hline
\end{tabular}

Ao: aortic root; IVS: interventricular septum; PW: posterior wall; LVdD: left ventricle diastolic diameter; LVsD: left ventricle systolic diameter; CMI: Cardiac Mass Index; E: E wave; A: A wave, EF: ejection fraction; LA: left atrium; RV: right ventricle; CMI: Cardiac Mass Index; T/R: thickness/radius; IVRT: isovolumic relaxation time; DTc: deceleration time; E/A: E peak/A peak ratio; $\mathrm{E}^{1}$ velocity; $\mathrm{A}^{1}$ velocity.

\subsection{Cardiopulmonary Test Parameters of RTR}

Aerobic and anaerobic thresholds of RTR were within the lower limit of the normal range at the beginning of the study. After six months of training, the $\mathrm{VO}_{2}$ peak and aerobic threshold had increased despite not being significantly so; while the anaerobic thresholds, estimated as the $\mathrm{VO}_{2}$ max level, were significantly higher. Blood pressure values, measured in resting condition, remained within normal values in RTR at different times of training : at the beginning(T0) the systolic blood pressure was $130.12 \pm 19.7$;sixth months later (T6) the diastolic blood pressure was $82.60 \pm 8.8$ ) in RTR , as well as in controls (T0 systolic blood pressure $135.15 \pm 18.6$; T6 diastolic blood pressure $80.50 \pm 9.8$ ).

\subsection{Echocardiographic Parameters}

All of the morphological and functional echocardiographic parameters were within the normal range, at the onset of the study and after six months of training. 
Some variations were found for the ventricular mass and wall thickness, despite not being significant, as well as a trend toward a reduction in LV diameters was observed. In a different manner, the EF had improved significantly after six months of training. Diastolic parameters were compatible with a mild dysfunction, and no modifications were observed after the exercise program. This aspect has been confirmed by Tissue Doppler Image (TDI) parameters.

\subsection{Strain Parameters}

All of the values of the LV GLS found in the transplants recipients were normal with respect to the validated values reported in the literature. The images were in any case at a good resolution for the post-processing strain analysis (Figure 1). Especially for the segmental strain values, the comparison with the data obtained from the healthy subjects' group, submitted to the same kind of exercise and for the same time, did not show, after six months of exercise, any statistical difference with an exclusion of the basal level of the LV chamber, where the values tend to be lower if compared to controls (Table 3). Particularly, the Lo Strain of the basal septum is, in RTR, significantly less reduced than the others.

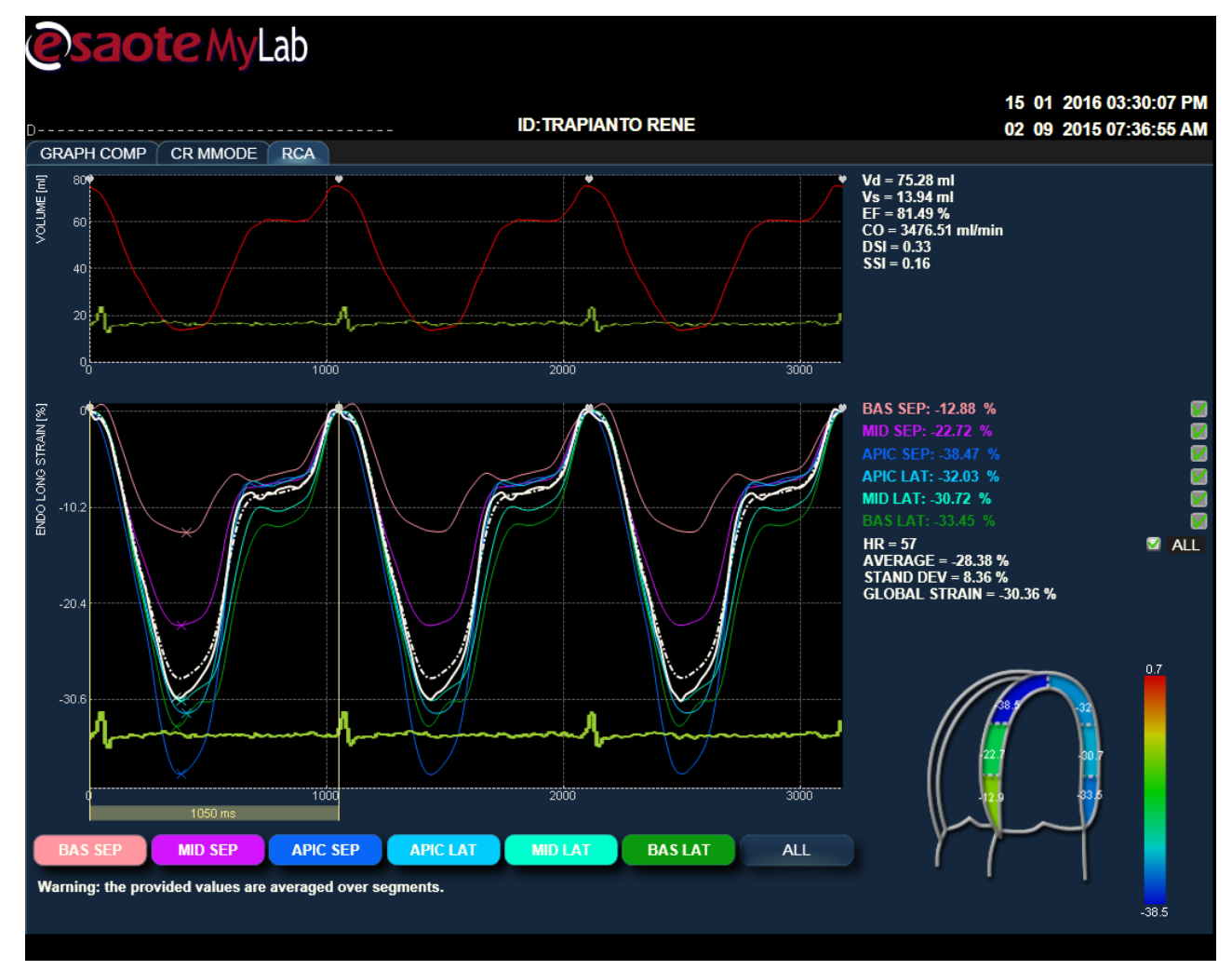

Figure 1. Strain analysis (XStrain Esaote) averaged from three cycles of cardiac HR. The image reproduces the summary of the strain analysis.

Table 3. Comparison of GLS and LV segmental values of the renal transplant recipients vs. controls after 6 months of training (T6).

\begin{tabular}{ccccccc}
\hline & LV-GLS & $\begin{array}{c}\text { LoS } \\
\text { Medium }\end{array}$ & Bas Sept & Bas Lat & Ap Lat & Ap Sept \\
\hline RTR & $-19.2 \pm 5.1$ & $-19.7 \pm 5.3$ & $-13.2 \pm 4.1$ & $-16.6 \pm 5.5$ & $-21 \pm 2.3$ & $-25.7 \pm-7.0$ \\
Controls & $-22.7 \pm-2.6$ & $-21.4 \pm 2.3$ & $-20.2 \pm 3$ & $-17.9 \pm 2.7$ & $-23.2 \pm 5.7$ & $-25.4 \pm 6$ \\
$p$ & NS & NS & 0.02 & NS & NS & NS \\
\hline
\end{tabular}

RTR: renal transplant recipients; LV-GLS: left ventricle global longitudinal strain; Bas Sept: basal septal; Bas Lat: basal lateral; Ap Lat: apical lateral; Ap Sept: apical septal. 


\section{Discussion}

Renal transplantation is a very common procedure in terminal kidney disease [6,21]. Patients do not often quickly resume a normal active lifestyle after surgery. This fact points to the need for greater attention to be paid to these subjects, particularly regarding their global management. Sedentary behavior and reduced daily physical activity represent the basic underlying causes for progressive worsening of those cardiovascular risk factors related to limited survivorship and increased morbidity [22]. It has been widely demonstrated that physical training improves particularly those cardiovascular risk factors strongly associated with a high risk of disability and death [1,21], especially if performed at moderate intensities [6,21]. Proper exercise can be however a complicated context considering the presence of many cardiovascular risks factors often present in an associated form as in the case of post-transplant syndrome. Strain analysis represents actually a current method for an adequate follow-up in many primary and secondary cardiac diseases $[9,10]$, where the standard evaluation by echocardiography cannot identify the eventual early dysfunction in asymptomatic subjects. An advantage to this condition, GLS in 2D-ST has been proven as a validated method to have clinical values to detect early contractile changes in the presence of preserved $\mathrm{EF}[9,10]$. From the results obtained, GLS and, especially, the LV segmental values can be used as a ready method to assess and to follow up on myocardial function. Potentially, current use of this method in this new field of interest of exercise as medicine can be considered. However, the reproducibility of the data presumes inevitably the high quality of the echo image, and for the validation of the results, it needs also a larger cohort of the population studied. Particularly regarding the echocardiographic parameters, the results support the importance of the GLS to follow the positive effect of this level of exercise in maintaining normal values of the systolic and diastolic LV function, with preserved and progressive enhanced EF after training. This is in agreement with the AT values found, as a parameter associated with increased survivorship, reduced mortality and morbidity [4,23]. The data are in fact significantly higher with respect to the initial phase of the study. Any possible further considerations of the trend toward a lower value of the LV strain values at the basal segments of the chamber, particularly if compared to the results of the control group, is not possible in this context. We do not know if this aspect make sense in a wider population of transplant recipients; therefore, a final interpretation of this behavior is not yet possible at this moment, and only a possible slight dysfunction can be hypothesized. This is one of the limits of the present study, due to the small group of subjects investigated in terms of the high level of the image resolution. Despite these boundaries, the principal message of the present study should be to highlight the feasibility and the potential clinical application of the strain analysis to follow the multiple effects of mixed exercise in post-renal transplantation. Further studies will be necessary to verify the efficacy of this exercise over a long-term period in order to permit to use Speckle Tracking (ST)evaluation as a tool used in daily practice for clinical decision-making.

Author Contributions: Stefani Laura conceived of, elaborated the echo data of and wrote the study. Gianni Pedrizzetti and Stefano Pedri revised the data and contributed to the management of the program. Marco Mandoli and Benedetta Tosi elaborated the data. Galanti Giorgio and Enrico Minetti revised and approved the final version of the study.

Conflicts of Interest: The authors declare no conflict of interest.

\section{References}

1. Glicklich, D.; Vohra, P. Cardiovascular risk assessment before and after kidney transplantation. Cardiol. Rev. 2014, 22, 153-162. [CrossRef] [PubMed]

2. Teixeira, A.P.; Fernandes, N.M.; Mata, G.F.; Chaoubah, A.; Paula, R.B.; Bastos, M.G. Prevalence of metabolic syndrome and its associated factors in renal transplant recipients. J. Bras. Nefrol. 2012, 34, 16-21. [PubMed]

3. Mikolasevic, I.; Racki, S.; Lukenda, V.; Pavletic-Persic, M.; Milic, S.; Orlic, L. Non-alcoholic fatty liver disease; a part of the metabolic syndrome in the renal transplant recipient and possible cause of an allograft dysfunction. Med. Hypotheses 2014, 82, 36-39. [CrossRef] [PubMed] 
4. Fellstrom, B.; Holdaas, H.; Jardine, A. Functional cardiopulmonary exercise testing in potential renal transplant recipients. J. Am. Soc. Nephrol. 2014, 25, 8-9. [CrossRef] [PubMed]

5. Galanti, G.; Stefani, L.; Mascherini, G.; Petri, C.; Corsani, I.; Francini, L.; Cattozzo, A.; Gianassi, M.; Minetti, E.; Pacini, A.; et al. Short-term prospective study of prescribed physical activityin kidney transplant recipients. Int. Emerg. Med. 2016, 11, 61-67. [CrossRef] [PubMed]

6. Roi, G.S.; Stefoni, S.; Mosconi, G.; Brugin, E.; Burra, P.; Ermolao, A.; Granito, M.; Mancini, P.; Mastrosimone, S.; Nacchia, F.; et al. Physical activity in solid organ transplant recipients: Organizational aspects and preliminary results of the Italian project. Transpl. Proc. 2014, 46, 2345-2349. [CrossRef] [PubMed]

7. De Luca, A.; Stefani, L.; Pedrizzetti, G.; Pedri, S.; Galanti, G. The effect of exercise training on left ventricular function in young elite athletes. Cardiovasc. Ultrasound 2011, 9. [CrossRef] [PubMed]

8. Stefani, L.; de Luca, A.; Maffulli, N.; Mercuri, R.; Innocenti, G.; Suliman, I.; Toncelli, L.; Vono, M.C.R.; Cappelli, B.; Pedri, S.; et al. Speckle tracking for left ventricle performance in young athletes with bicuspid aortic valve and mild aortic regurgitation. Eur. J. Echocardiogr. 2009, 10, 527-531. [CrossRef] [PubMed]

9. Claus, P.; Omar, A.M.; Pedrizzetti, G.; Sengupta, P.P.; Nagel, E. Tissue tracking technology for assessing cardiac mechanics. Principles, normal values and clinical application. JACC Cardiovasc. Imaging 2015, 8 , 1444-1460. [CrossRef] [PubMed]

10. D’hooge, J.; Barbosa, D.; Gao, H.; Claus, P.; Prater, D.; Hamilton, J.; Lysyansky, P.; Abe, Y.; Ito, Y.; Houle, H.; et al. Two-dimensional speckle tracking echocardiography: Standardization efforts based on synthetic ultrasound data. Eur. Heart J. Cardiovasc. Imaging 2015. [CrossRef] [PubMed]

11. Farsalinos, K.E.; Daraban, A.M.; Ünlü, S.; Thomas, J.D.; Badano, L.P.; Voigt, J.U. Head-to-head comparison of global longitudinal strain measurements among nine different vendors: The EACVI/ASE inter-vendor comparison study. J. Am. Soc. Echocardiogr. 2015, 28, 1171-1181. [CrossRef] [PubMed]

12. Lang, R.M.; Badano, L.P.; Mor-Avi, V.; Afilalo, J.; Armstrong, A.; Ernande, L.; Flachskampf, F.A.; Foster, E.; Goldstein, S.A.; Kuznetsova, T.; et al. Recommendations for cardiac chamber quantification by echocardiography in adults: An update from the American Society of Echocardiography and the European Association of Cardiovascular Imaging. J. Am. Soc. Echocardiogr. 2015, 28, 1-39. [CrossRef] [PubMed]

13. Chodzko-Zajko, W.J.; Proctor, D.N.; Fiatarone Singh, M.A.; Minson, C.T.; Nigg, C.R.; Salem, G.J.; Skinner, J.S. Position stand. Exercise and physical activity for older adults. Med. Sci. Sports Exerc. 2009, 41, 1510-1530. [CrossRef] [PubMed]

14. Brzycki, M. A Practical Approach to Strength Training; McGraw-Hill: New York, NY, USA, 1998.

15. Voigt, J.U.; Pedrizzetti, G.; Lysyansky, P.; Marwick, T.H.; Houle, H.; Baumann, R.; Pedri, S.; Ito, Y.; Abe, Y.; Metz, S.; et al. Definitions for a common standard for 2D speckle tracking echocardiography: Consensus document of the EACVI/ASE/Industry Task Force to standardize deformation imaging. Eur. Heart J. Cardiovasc. Imaging 2015, 16, 1-11. [CrossRef] [PubMed]

16. Gibbons, R.J.; Balady, G.J.; Bricker, J.T.; Chaitman, B.R.; Fletcher, G.F.; Froelicher, V.F.; Mark, D.B.; McCallister, B.D.; O’Reilly, M.G.; Winters, W.L., Jr.; et al. American College of Cardiology/ American Heart Association Task Force on Practice Guidelines (Committee to Update the 1997 Exercise Testing Guidelines). ACC/AHA 2002 guideline update for exercise testing: Summary article: A report of the American College of Cardiology / American Heart Association Task Force on Practice Guidelines (Committee to Update the 1997 Exercise Testing Guidelines). Circulation 2002, 106, 1883-1892. [PubMed]

17. Ghosh, A.K. Anaerobic threshold: Its concept and role in endurance sport. Malays. J. Med. Sci. 2004, 11, 24-36. [PubMed]

18. Robertson, R.J.; Goss, F.L.; Dubé, J.; Rutkowski, J.; Dupain, M.; Brennan, C.; Andreacci, J. Validation of the adult OMNI scale of perceived exertion for cycle ergometer exercise. Med. Sci. Sports Exerc. 2004, 36, 102-108. [CrossRef] [PubMed]

19. Douglas, P.S.; Garcia, M.J.; Haines, D.E.; Lai, W.W.; Manning, W.J.; Patel, A.R.; Picard, M.H.; Polk, D.M.; Ragosta, M.; Ward, R.P.; et al. ACCF/ ASE/ AHA/ ASNC/HFSA/ HRS/ SCAI/ SCCM/ SCCT/ SCMR 2011 Appropriate Use Criteria for Echocardiography. J. Am. Coll. Cardiol. 2011, 57, 1126-1166. [CrossRef] [PubMed]

20. Devereux, R.B. Detection of left ventricular hypertrophy by M-mode echocardiography. Anatomic validation, standardization, and comparison to other methods. Hypertension 1987, 9, II19-II26. [CrossRef] [PubMed] 
21. Riess, K.J.; Haykowsky, M.; Lawrance, R.; Tomczak, C.R.; Welsh, R.; Lewanczuk, R.; Tymchak, W.; Haennel, R.G.; Gourishankar, S. Exercise training improves aerobic capacity, muscle strength, and quality of life in renal transplant recipients. Appl. Physiol. Nutr. MeTable 2014, 39, 566-571. [CrossRef] [PubMed]

22. Sui, X.; Laditka, J.N.; Hardin, J.W.; Blair, S.N. Estimated functional capacity predicts mortality in older adults. PED. J. Am. Geriatr. Soc. 2007, 55, 1940-1947. [CrossRef] [PubMed]

23. Bozbas, H.; Altin, C.; Karacaglar, E.; Kanyilmaz, S.; Yildirir, A.; Muderrisoglu, H.; Haberal, M. The prevalence and types of cardiovascular disease in patients with end-stage renal disease undergoing renal transplantation. Transpl. Proc. 2013, 45, 3478-3480. [CrossRef] [PubMed]

(C) 2016 by the authors; licensee MDPI, Basel, Switzerland. This article is an open access article distributed under the terms and conditions of the Creative Commons by Attribution (CC-BY) license (http://creativecommons.org/licenses/by/4.0/). 